\title{
Challenges and Opportunities Associated with Whole Grains Use in Twin Cities Restaurants: A Food Systems Perspective
}

\author{
Keagan Ringling ${ }^{1}$, Alexander Yangas ${ }^{1}$, Melissa Riley $^{1}$, Renee Korczak $^{1} \&$ Len Marquart $^{1}$ \\ ${ }^{1}$ Department of Food Science and Nutrition, University of Minnesota, USA \\ Correspondence: Len Marquart, Department of Food Science and Nutrition, University of Minnesota, USA. \\ E-mail: 1marquar@umn.edu
}

Received: April 30, 2019

Accepted: May 17, 2019 Online Published: June 16, 2019

doi:10.5539/jfr.v8n4p44

URL: https://doi.org/10.5539/jfr.v8n4p44

\begin{abstract}
Increasing whole grain (WG) availability in restaurants allows consumers to make healthier choices with minimal effort while improving adherence to the Dietary Guidelines. To understand challenges associated with increasing WG availability in Twin Cities (Minneapolis \& St. Paul, Minnesota, USA) restaurants, interviews and focus groups were conducted with 24 local, national, and international food system members. This report identifies food system barriers, including policy, business, and societal pressures, that limit availability of WG based foods in restaurants. Insight provided by this study allows consortium members from various sectors and disciplines to work on a precompetitive basis to include more WG in Twin Cities restaurants.
\end{abstract}

Keywords: whole grain, supply chain, systems nutrition, menu options

\section{Introduction}

Food environments influence diet and health. These environments are the human-built and social environments that include physical, social, economic, cultural, and political factors that impact accessibility, availability, and adequacy of food within a community or region (Rideout et al., 2015). For example, consumer food environments are characterized by the availability, variety, price and quality of foods as well as promotional signs and prominence of healthful versus non-healthful food options (Rideout et al., 2015). Restaurants provide one example of a consumer food environment that has a major influence on diet and health.

As consumers continue to rely on restaurants for food, their intake becomes dependent on available menu options. Restaurant meals have been shown to be generally less healthful as these meals provide more calories, total and saturated fat, sodium, and cholesterol per meal than a typical meal prepared at home (Scourboutakos \& L'Abbe, 2012; Hearst et al., 2013). Conversely, meals away from home (AFH) can be low in several key nutrients, including fiber, calcium, and iron (Lendway \& Hesse, 2014). Research indicates that AFH eating is associated with increased biomarkers of chronic disease (Kant et al., 2015). Indeed, as the reliance on AFH eating increases, it becomes clear that restaurants must play a role in offering and promoting healthier meal options.

Given the current consumer food environment, actions to improve the nutritional quality of menu options in restaurants remains prudent. Substituting WG's for refined grain products can increase the nutritive value of foods without drastically changing acceptability and organoleptic qualities of meals (Rosen et al., 2008). The most common grains in the American diet include wheat, oats, rice, maize, and rye. These grains in their whole form contain an abundance of nutrients, including vitamin E, magnesium, iron, fiber, several B vitamins, and other beneficial phytochemicals (Liu, 2007). Diets containing WG's are inversely related with obesity, insulin resistance, diabetes, and inflammation (Lutsey et al., 2007). Whole grain consumption is also associated with reduced risk of coronary artery disease, cardiovascular disease, and total mortality (Huang et al., 2015). For these reasons, the 2010 and 2015 Dietary Guidelines for Americans recommend that at least half of all grain servings should be WG (U.S. Department of Health and Human Services [USDHHS] and U.S. Department of Agriculture [USDA], 2010; 2015). More specifically, the Guidelines recommend that all adults and children over 9 years consume at least 3 servings of WG's per day. However, data from the United States Department of Agriculture (USDA) showed that Americans only consumed an average of 0.90 servings of WG's per day in 2013-2014 (Bowman et al., 2017) Results from the 2009-2010 National Health and Nutrition Examination Survey (NHANES) indicated that only $2.9 \%$ of children and adolescents (2-18 years old) and $7.7 \%$ of adults (over 18 
years old) met the recommended intake of WG's (Reicks et al., 2014). Increasing the availability of WG based foods in restaurants could allow consumers to make healthier choices with minimal knowledge and effort while also improving their adherence to the Dietary Guidelines.

Currently, barriers exist within the food system and food environment that prevent the incorporation and increased availability of WG based foods in restaurants. These barriers include limited knowledge regarding WG foods, undesirable taste or texture qualities, increased preparation time, higher cost, and low availability in foodservice settings (Rosen et al., 2012). Traditionally, the responsibility has fallen on consumers to manage their health by actively seeking nutritious foods when making dietary choices. However, several studies indicate that the current consumer food environment obstructs, rather than supports, healthy eating habits (Harvard School of Public Health, 2015). For this reason, our study focused on factors across the food supply that limit whole grain use in Twin Cities (Minneapolis and St. Paul) restaurants beyond consumer and restaurateur preferences. A systems perspective demonstrates that, while the ultimate decision of whether to consume WG's lies with the consumer, the circumstances which most greatly influence that decision are pre-determined long before the consumer reaches the restaurant. The findings of this study may elucidate ways to utilize the supply chain and restaurant settings to make healthier, WG menu options a more effortless and instinctive choice for consumers when eating out.

\section{Methods}

The Institutional Review Board (IRB) at the University of Minnesota approves this study. Individual interviews $(n=24)$ were conducted during the summer of 2013 with a convenience sample of local, national, and international food system members that contribute to the Twin Cities restaurants. Individual interview participants included culinary $(n=4)$, baking $(n=2)$, milling $(n=5)$, food supply chain $(n=7)$, public health $(n=2)$, and academic professionals $(n=4)$. Participants were selected from throughout the WG food system so researchers could understand a broad a range of perspectives. Most interviews were 45 minutes to one hour in duration. Interviews were generally informal and allowed for deviation from the original interview questions. This method was chosen to understand broad themes related to different sectors and disciplines involved in the WG food system. Sessions were audiotaped and transcribed verbatim. Two investigators then independently coded transcripts to generate themes and ascribe them to the appropriate tiers of an Adapted Social-Ecological Model (ASEM) (Richard et al., 2011).

During interviews, participants were asked questions related to the following: (1) The participant's role in influencing WG consumption; (2) The influence of the grain supply chain on WG consumption in restaurants; (3) The influence of public health initiatives on WG consumption in restaurants; (4) The specific barriers preventing WG availability and consumption in restaurants; (5) Potential strategies for increasing WG availability and consumption in restaurants; and (6) Intra-sector and inter-sector collaboration between the participant and other professionals.

\subsection{The Adapted Social-Ecological Model}

The Adapted Social-Ecological Model (ASEM) is a five-tier modification of what is traditionally a four-tiered model (Richard et al., 2011). The Social-Ecological Model includes the following tiers: Individual, Interpersonal, Built Environment, and Policy/Society. In the ASEM, the "Built Environment" tier is subdivided into "Restaurants" and "Supply Chain." The ASEM more accurately represents the relationship between the two subcategories and the end consumer. Within the context of AFH eating, restaurants have direct influence on consumer choices, while the supply chain typically has an indirect influence through the restaurants (menu options). The ASEM demonstrates how consequences of the supply chain/restaurant relationship, which would be lost in a traditional model, have tremendous influence on consumer behavior (Richard et al., 2011). Moreover, the adoption of WG presents issues in restaurants, which would not apply upstream in the supply chain, and vice-versa. This model preserves the unique needs of each tier, while demonstrating their interconnectedness and cascading effects on the end consumer.

\section{Results}

Several themes were identified from participant responses. These themes have been categorized according to the applicable tiers of the ASEM. The themes and challenges in each tier of the ASEM will be addressed in ascending order, beginning at the individual level, and culminating with Policy/Societal influence. General themes related to participant beliefs about opportunities are also presented for each ASEM tier.

\subsection{Individual}

Interview evaluations revealed four major barriers to WG consumption in restaurants on the individual level. 
These themes included: (1) health stigma, (2) sensory characteristics, (3) cost, and (4) availability, as a majority of participant responses focused on the individual level.

\subsubsection{Health Stigma}

Eleven respondents indicated the common perception of WG as a "health food" hinders consumption in restaurants. Although most participants acknowledged that some people seek WG when eating out, the majority is discouraged due to its reputation as "health food." "WG can easily be stigmatized as "healthy" and healthy is death for consumer demand in many circles (Academic)." This mentality was reflected well by one quote from a public health professional who commented, "Some people go specifically to indulge themselves in restaurants, and don't want to be reminded that they're eating things that aren't necessarily the best for them...people want to indulge, and they see WG not as an indulgence, but as something that isn't as good." An academic expressed a similar sentiment, saying, "I think to a certain extent, sometimes people get saturated with the health message. And so they may have a demand, but they don't want to be told all the time that they have to be healthy."

\subsubsection{Sensory Characteristics}

Seven respondents indicated that unpleasant taste or textural characteristics in WG foods are a significant deterrent to their appeal with consumers. Whole grain products contain compounds that are not present in their refined counterparts. These compounds alter the taste of the final product, as well as its texture and color. Whole grain products have been described as "bitterer", "denser", "heavier" and "earthier" tasting than the refined version of the same product. Yet, there was little interest and/or motivation by respondents to want to overcome challenges and seek opportunities to develop and deliver WG foods that consumers desire and want to eat.

\subsubsection{Cost}

Six respondents indicated that the increased costs associated with WG foods prevent their consumption in restaurants. Whole grain products are often considered premium items due to the relatively low demand for these products and the difficulty of procuring them on a large scale. Until the demand for WG's reaches a critical mass, the supply will constantly be insufficient to meet the needs of those who require the products in greater volumes, such as restaurants. Whole grain products have a shorter shelf life than refined grain products, which limits the time it can spend in distribution centers or warehouses. Thus, most distributors only acquire the quantity necessary to meet current customer demand. This demand is often low because restaurants must pay a premium to acquire many WG products in the quantities necessary to serve their clientele. This cost is invariably passed on to the consumer who may or may not even be willing to try the dish. Restaurants will not sacrifice storage space, equipment, and profits for a product that may sit on a shelf until it expires, or worse, may alienate their customers. Rather than take this risk, a restaurateur may opt for the cheaper refined grain option that is more likely accepted by the majority.

\subsubsection{Availability of Added Value Grain-Based Foods}

Several respondents indicated that low availability of quality WG prevented widespread usage in restaurants. Frequently voiced opportunities included increasing the availability of popular or frequently consumed foods in restaurants that can be made with WGs that are healthier, cost-effective and desirable. These WG foods would be readily accessible to restaurant clientele and easy for chefs and food service personnel to purchase, prepare and serve in an efficient and economical manner. Children were also mentioned as a primary demographic targeted for greater exposure to WG foods.

\subsection{Interpersonal}

Few people communicated or initiated efforts related to WG's within the supply chain and food environment. Millers and bakers commented that a majority of their work revolves around communication related to refined grain flour and their respective baked products. Overall, participant responses about communication and collaboration were less frequently voiced relative to the use of WG's. From a chef's point of view, "The only collaboration I have about WG's is people want me to use their ingredients, so I can sell it to the customer." This may indicate a lack of support in restaurants to help chefs succeed using WG ingredients and WG foods that restaurant clientele will eat. On the other hand, it was voiced "People [Patrons] don't really have the venue to make their preferences known in restaurants." Participants suggested opportunities may exist to better understand the value and meaning of WG's relative to supply chain players, restaurant owners, managers and clientele. A deeper examination of the potential roles, functions and activities of these players might help gauge more appropriate use of WG foods in restaurants. 


\subsection{Restaurants}

Participants reported that chefs and the general public are often intimidated by new experiences like preparing, serving, and eating WG. Other participants indicated restaurants have no immediate incentive to use WG, thus "Higher quality ingredients do not necessarily equal higher quality products." These ingredients can also be more expensive with no guaranteed reward. As a means to counter this lack of skill and hands-on experience most participants suggested chefs, cooks and restaurant staff need more training. Currently, there are few to no dollars spent teaching people how to work with WG. As a result, chefs, cooks, and restaurant staff lack foundational skills in purchasing, preparing, storing and serving these foods. Ultimately, the limited foundation through the food industry, consumers, chefs, and others inhibits whole grain adoption. Thus, a majority of participants suggested considerable benefit might result in training restaurant chefs, kitchen personnel, and serving staff in purchasing, storing, preparing and marketing popular foods made with WG.

\subsubsection{Supply Chain}

Study participants with primary roles within the supply chain stated, “...the supply chain is engineered to produce refined products in large quantities, unlike WG." As mentioned by a wheat breeder, "wheat is bred for optimally refined grain foods, while flour quality is based on refined grain product quality and yield, not for WG foods." From a miller's perspective, there are unique processing needs for WG. In some instances, WG milling requires capital investments in new equipment, such as additional storage silos and milling technology. It was stated strongly that academic interests and research priorities are seldom aligned with industry or consumer priorities. Thus, one food scientist indicated a tremendous need for cross-sector and cross-disciplinary training whereby faculty spend time in industry while industry scientists collaborate more intimately with universities. The intent was to increase understanding and appreciation for collaborative efforts around healthier grain-based foods. Systematically mapping barriers and opportunities in the development, delivery, and consumption of WG foods would allow for a more comprehensive look at the gaps in the supply chain, food environment, and community relative to WG use in restaurants. Additional insight from this effort could provide a means for prioritizing precompetitive and relevant research and education focus.

\subsubsection{Policy / Societal:}

From a policy and societal perspective white bread is ingrained in American culture. Most participants commented they engage in a greater frequency of refined grain activities compared to WG's across the sectors and disciplines within the food system. Overall, most activities were reactive (e.g. creating press releases against low carb, gluten free, etc.) rather than promoting the health attributes of grains through the inclusion of WG and fiber in frequently consumed foods.

WG's are not nearly as available through distributors in comparison to refined grains. For example, McDonald's tried to offer a whole wheat bun once, but stopped because customers preferred a white bun. From the perspective of general societal understanding of WG, one industry representative said, "whole grain definitions are too ambiguous and confusing. Consumers don't understand what WG means and neither do the chefs cooking in restaurants."

Based on participant input, opportunities may exist through a unified inter-sector collaboration by developing and implementing a comprehensive public health campaign to build positive messaging around WG foods. Focus would be encouraged through education about WG in harmony with greater availability of WG foods in the marketplace.

\section{Discussion}

This study examined current roles and perceptions of supply chain members, along with challenges and opportunities that each member experienced in bringing WG's from field to fork. Objectives were based on the 2010 Dietary Guidelines which asked for new food introductions and reformulation of prepared foods that deliver good taste and convenience while meeting dietary recommendations and cost constraints (USDHHS \& USDA, 2010; 2015).

The food environment remains mostly devoid of viable WG choices for most Americans, as consumers fail to meet the Dietary Guidelines recommendations which state "at least half of all grain servings should be WG's" (USDHHS \& USDA, 2010; 2015). Study participants consistently echoed an overarching theme, that there is little translation of agricultural food policy and dietary guidance into easy access to WG foods, which allows consumers to eat more. From a historical perspective, the grain industry is designed to proliferate the continued milling and sourcing of refined grain ingredients for baking and food manufacturing. In contrast, the WG infrastructure lacks the tools, approaches, methods, foundational knowledge, and standard business practices 
necessary to carry healthful WG foods from field to consumer. The refined grain system is generally more efficient and cost effective to source, process, market and profit by selling refined grain foods that are practical, affordable and desirable for consumers. Based on this sample of participants, there appears to be a lack of shared value and meaning around WG food-related collaboration and communication strategies.

Barriers to using WG's occur throughout the supply chain from growing the grain (i.e. red wheat bred for refined grain use, lack of incentives to grow white wheat; pre-sprouting of white wheat places profits at risk), milling of grain into WG ingredients (different particle sizes result in varying functionalities, more enzymatic activity, lipids, and antioxidants which effect end-use and storage properties), baking and manufacturing into foods (lack of standard approaches and methods for WG product development; shorter shelf-life and storage), distributing and purchasing (lack of WG volume, distribution channels and cost constraints), preparing and serving in a restaurant setting (chef, production and serving staff are unfamiliar with WG ingredient storage, WG food preparation, and service) and consumer needs, wants and desires (lack of availability and accessibility of tasty added value WG foods). Until the necessary tools, approaches and WG infrastructure are established and leveraged in an intentional, synchronous and consistent manner throughout the grains supply chain and food environment, there is little motivation for players in the supply chain to support, encourage or use WG ingredients.

Restaurants are encouraged to gradually shift to incorporate more WG menu options so that AFH consumers can come closer to meeting WG recommendations. Perhaps a gradual increase in the availability of WG foods in schools and in restaurants is one approach to increasing consumption. For example, "sneaking in" better-for-you WG foods in school cafeterias and in restaurants without identifying it on the menu has the potential to be successful. Small recipe substitutions often go unnoticed, and some food and nutrition service directors already do this in hospital cafeterias. This practice is known as "stealth health", and it is a way to improve the nutritional quality of foods without customers even noticing (Food Service Director, 2012).

An example of increasing WG's in the food supply is to reformulate a popular food product such as pizza. Previous research has shown that modifications to pizza crust to include WG flour have been made without affecting acceptance among school children (Chan et al., 2008). This type of pizza is currently available for school meals but has limited availability in other food service and retail markets (Schwan's, 2013). Pizza can also be reformulated to be WG rich, lower sodium, and lower fat. Since pizza is made up of different food groups and is widely consumed and accepted; it has the potential to be a healthier staple that targets acceptable levels of WG's, vegetables, and dairy with lower fat and sodium. Pizza is just one way that foods can be modified to more closely meet Dietary Guidelines recommendations while satisfying consumer expectations for cost, taste and convenience (Jacques et al., 2013).

Efforts to gradually introduce more WG's into the marketplace provide new, cutting edge opportunities for collaboration throughout the grains community. Cross-disciplinary and cross-sector collaborations will help facilitate progress toward novel approaches to research, discovery, development, and delivery of WG based foods while collectively solving major gaps in the WG supply chain and food environment. Priorities and focus might be established on a precompetitive basis among the sectors and disciplines, related to the type of grain and food product, and place of service in the community, such as introducing WG pizza into restaurants (Tritt et al., 2015). To accomplish this system-wide goal, it requires synchronizing individual (players) and organizational contributions around a grand challenge resulting in collective impact. Adopting a culture of systems thinking while reconfiguring tools, approaches, and food design to emphasize shared value for profit and health can allow the grains community to overcome limitations in the current paradigm.

Scientists must continue to conduct research on WG's and health to solidify the knowledge base, while industry has the unique opportunity to reformulate products to make them healthier (McKeown et al., 2013). Examples of tools, techniques, and methods that might be developed include analytical methods for determining particle size, stability and rancidity in WG ingredients and foods. National and global institutions such as the International Association of Cereal Science and Technology (ICC) can play a major role in allowing these methods and techniques to be available to cereal scientists, product developers and bakers. Standard methods for using WG ingredients in grain-based foods such as bread, tortillas, pasta, cereals, and other baked products provide a precompetitive base for all bakers and manufacturers to develop and deliver better WG foods.

\subsection{Study Limitations and Strengths}

We acknowledge the limitations and strengths of this research. One of the limitations of this study was that a convenience sample was used to assess the barriers that exist within the food system and food environment to incorporate WG foods in restaurants. The sample size $(n=24)$ was also small which prevents the generalization of 
results on a large scale. Finally, one of the other major drawbacks to a convenience sample is the opportunity for bias to cloud the results. In terms of strengths, even though the sample was small, the individuals surveyed represented different sectors of the food system (i.e. academics, non-profit organizations, public health, government, and industry). Having perspective from these diverse disciplines is critical for developing practical solutions to deliver WG's and WG foods into the food supply.

Although the methods are not fully objective the intent of the paper was to show the 'big-picture' interrelationships amongst the roles, functions, and activities that take place throughout the whole grain food system.

\section{Conclusion}

Strategies to increase WG intake in the US diet should target action at each level within the food system and food environment, such as in restaurants. Restaurateurs are apprehensive in the purchase, preparation, and service of WG foods. This may be attributed to unfamiliarity, insufficient demand, shorter shelf life, and potential loss of profit. A WG/health stigma interferes with restaurant clientele selection and consumption of WG foods. Consumers are looking for WG foods that are affordable, and thus, readily available. Although incorporating WG's into restaurants may not solve all of these health problems, it is a step in the right direction, especially for young children who can establish healthy eating patterns at an early life stage. Based on this assessment, we will continue to work with a consortium of members from various sectors and disciplines to identify challenges and opportunities for the inclusion of more WG's in the Twin Cities (Minneapolis and St. Paul). The question remains: How do we create a supply chain and food environment that supports a culture of developing, delivering and increasing consumption of WG foods where interdependent stakeholders can perform their individual roles and effectively collaborate with each other? In part, added value for grain-based foods lies in the strategic use of WG flour to carefully achieve refined to WG flour ratios in most foods that are practical, healthier, affordable and desirable.

\section{References}

Bowman, S. A., Clemens, J. C., Friday, J. E., Lynch, K. L., LaComb, R. P., \& Moshfegh, A. J. (2017). Food Patterns Equivalents Intakes by Americans: What We Eat in America. NHANES 2003-04 and 2013-14. Food Surveys Research Group. Dietary Data Brief No. 17. https://www.ars.usda.gov/ARSUserFiles/80400530/pdf/DBrief/17_Food_Patterns_Equivalents_0304_1314. pdf

Chan, H. W., Burgess Champoux, T., Reicks, M., Vickers, Z., \& Marquart, L. (2008). White whole-wheat flour can be partially substituted for refined-wheat flour in pizza crust in school meals without affecting consumption. Journal of Child Nutrition and Management, 32(1).

Chase, K., Reicks, M., Srinvasan, P., \& Marquart, L. (2004). Perceptions of members of the American Association of Cereal Chemists regarding production of whole grain foods. Journal of Food Distribution Research, 35(856-2016-56646), 53.

Food and Drug Association. (2006). Guidance for Industry and FDA Staff Whole Grain. Retrieved from http://www.fda.gov/OHRMS/DOCKETS/98fr/06d-0066-gdl0001.pdf

Ferruzzi, M. G., Jonnalagadda, S. S., Liu, S., Marquart, L., McKeown, N., Reicks, M., ... van der Kamp, J. W. (2014). Developing a standard definition of whole-grain foods for dietary recommendations: summary report of a multidisciplinary expert roundtable discussion. Advances in nutrition, 5(2), 164-176. https://doi.org/10.3945/an.113.005223

Food Service Director. (2012). What is "stealth health?". Retrieved from https://www.foodservicedirector.com/operations/what-stealth-health

Harvard School of Public Health. (2015). Toxic Food Environment: How our Surroundings Influence What We Eat. Obesity Prevention Resource. Retrieved from https://www.hsph.harvard.edu/obesity-prevention-source/obesity-causes/food-environment-and-obesity

Hearst, M. O., Harnack, L. J., Bauer, K. W., Earnest, A. A., French, S. A., \& Oakes, J. M. (2013). Nutritional quality at eight US fast-food chains: 14-year trends. American journal of preventive medicine, 44(6), 589-594. https://doi.org/10.1016/j.amepre.2013.01.028

Huang, T., Xu, M., Lee, A., Cho, S., \& Qi, L. (2015). Consumption of whole grains and cereal fiber and total and cause-specific mortality: prospective analysis of 367,442 individuals. BMC medicine, 13(1), 59. https://doi.org/10.1186/s12916-015-0294-7 
Jacques, P., Hauge, D., Voth, K., Hermann, M., Maschoff, B., \& Marquart, L. (2013). Overcoming the challenges of translating the US dietary guidelines into healthier grain-based foods. Nutrition Today, 48(6), 254-259. https://doi.org/10.1097/NT.0000000000000004

Kant, A. K., Whitley, M. I., \& Graubard, B. I. (2015). Away from home meals: associations with biomarkers of chronic disease and dietary intake in American adults, NHANES 2005-2010. International journal of obesity, 39(5), 820. https://doi.org/10.1038/ijo.2014.183

Lendway, W., Hesse, D., \& Marquart, L. (2014). Minneapolis/St. Paul Chefs' Attitudes and Behaviors Regarding Whole Grain Use. Journal of foodservice business research, 17(2), 122-135. https://doi.org/10.1080/15378020.2014.902646

Lin, B. H., Guthrie, J., \& Frazao, E. (1999). Nutrient contribution of food away from home. America's eating habits: Changes and consequences, 750.

Liu, R. H. (2007). Whole grain phytochemicals and health. Journal of Cereal Science, 46(3), 207-219. https://doi.org/10.1016/j.jcs.2007.06.010

Lutsey, P. L., Jacobs, D. R., Kori, S., Mayer-Davis, E., Shea, S., Steffen, L. M., ... Tracy, R. (2007). Whole grain intake and its cross-sectional association with obesity, insulin resistance, inflammation, diabetes and subclinical CVD: The MESA Study. British Journal of Nutrition, 98(2), 397-405. https://doi.org/10.1017/S0007114507700715

McKeown, N. M., Jacques, P. F., Seal, C. J., de Vries, J., Jonnalagadda, S. S., Clemens, R., ... Murray, R. (2013). Whole grains and health: from theory to practice - highlights of the Grains for Health Foundation's Whole Grains Summit 2012. The Journal of nutrition, 143(5), 744S-758S. https://doi.org/10.3945/jn.112.172536

Reicks, M., Jonnalagadda, S., Albertson, A. M., \& Joshi, N. (2014). Total dietary fiber intakes in the US population are related to whole grain consumption: results from the National Health and Nutrition Examination Survey 2009 to 2010. Nutrition research, 34(3), 226-234. https://doi.org/10.1016/j.nutres.2014.01.002

Richard, L., Gauvin, L., \& Raine, K. (2011). Ecological models revisited: their uses and evolution in health promotion over two decades. Annual review of public health, 32, 307-326. https://doi.org/10.1146/annurev-publhealth-031210-101141

Rideout, K., Mah, C. L., \& Minaker, L. (2015). Food environments: an introduction for public health practice. National Collaborating Centre for Environmental Health British Columbia Centre for Disease Control: Vancouver, BC, Canada.

Rosen, R. A., Burgess-Champoux, T. L., Marquart, L., \& Reicks, M. M. (2012). Associations between whole-grain intake, psychosocial variables, and home availability among elementary school children. Journal of nutrition education and behavior, 44(6), 628-633. https://doi.org/10.1016/j.jneb.2010.02.007

Rosen, R. A., Sadeghi, L., Schroeder, N., Reicks, M. M., \& Marquart, L. (2008). Gradual Incorporation of Whole Wheat Flour into Bread Products for Elementary School Children Improves Whole Grain Intake. Journal of Child Nutrition \& Management, 32(2), $\mathrm{n} 2$.

Scourboutakos, M. J., \& L'Abbé, M. R. (2012). Restaurant menus: calories, caloric density, and serving size. American journal of preventive medicine, 43(3), 249-255. https://doi.org/10.1016/j.amepre.2012.05.018

Schwan's. (2013). Schwan's BIG DADDY'S Bold 16" Rolled Edge Cheese Pizza. Retrieved from http://www.schwans.com

Tritt, A., Reicks, M., \& Marquart, L. (2015). Reformulation of pizza crust in restaurants may increase whole-grain intake among children. Public health nutrition, 18(8), 1407-1411. https://doi.org/10.1017/S1368980014001724

United States Department of Agriculture ARS. (2012). Nutrient Intakes from Food: Mean Amounts Consumed per Individual, by Gender and Age, What We Eat in America, NHANES 2009-2010. Retrieved from https://www.ars.usda.gov/ARSUserFiles/80400530/pdf/0506/Table_1_NIF_05.pdf

U.S. Department of Health and Human Services and U.S. Department of Agriculture. 2010 - 2015 Dietary Guidelines for Americans. $7^{\text {th }}$ Edition. December 2010. Retrieved from https://health.gov/dietaryguidelines/2010/guidelines/ 
U.S. Department of Health and Human Services and U.S. Department of Agriculture. 2015 - 2020 Dietary Guidelines for Americans. $8^{\text {th }}$ Edition. December 2015. Retrieved from https://health.gov/dietaryguidelines/2015/guidelines/

\section{Copyrights}

Copyright for this article is retained by the author(s), with first publication rights granted to the journal.

This is an open-access article distributed under the terms and conditions of the Creative Commons Attribution license (http://creativecommons.org/licenses/by/4.0/). 